\title{
A Study on Transportation Algorithm of Bi-Level Logistics Nodes Based on Genetic Algorithm
}

\author{
Jiacheng $\mathrm{Li}^{1}$ and Lei $\mathrm{Li}^{1}$ \\ ${ }^{1}$ Faculty of Science and Engineering, Hosei University, Tokyo, Japan
}

\begin{abstract}
To study common transportation lines and transportation vehicle selection problem, the paper makes mathematical modeling against vehicle scheduling and transportation line in bi-level node transportation line based on genetic algorithm with the optimization objective of bi-level logistics node transportation expense after considering the allocation strategy between the path of vehicle picking up and delivering goods simultaneously and vehicle model with different paths. Applying MATLAB software, the paper solves the model based on traditional genetic algorithm and single parent genetic algorithm and verifies the correctness and effectiveness of the model and single parent genetic algorithm. It indicates that the model and algorithm proposed in the paper could solve bi-level node transportation problem of multiple vehicle models better.
\end{abstract}

Keyword : Isomerism vehicle, Transportation line, Parthenon-Genetic Algorithm

\section{Introduction}

Vehicle scheduling and selection of transportation line in the vehicle transportation is the key link of modern logistics system. It involves widely and needs to consider many factors. Moreover, it has important significance on enhancing service quality, reducing logistics cost and increasing economic benefit. Research on vehicle transportation scheduling traces back to 1959 that Dantzig and Ramser proposed vehicle transportation scheduling optimization and proposed corresponding mathematical planning model and its solution algorithm [1]. Subsequently, foreign and domestic scholars has made in-depth research and made many excellent achievements. Brank et al (2005) has studied the order change in the vehicle distribution process. Taking the longest online time of vehicle as the target, it optimized the vehicle path [2]. Li et al (2007) proposed rescheduling the reserve vehicle against the dynamic customer demand and established corresponding mathematical model of scheduling [3]. Wang Liang, Li Shiqiao (2007) have studied the stochastic distribution strategy of demand in the two-stage distribution system, proposed optimized integration of inventory control and transportation decision and established corresponding mathematics, but the driving circuit planning problem is not mentioned [4]. Yang Yanng et al (2001) has studied the optimized integration of inventory and distribution in the supply chain of distributor and retailer in dynamic environment, built the real-time scheduling model of mobile supply chain, and demonstrated the mechanism and effectiveness of mobile supply chain in reducing the cost [5]; Wang Xu et al (2012) has proposed the solution of using quantum genetic algorithm for solving vehicle routing and minimizing the distribution journey is superior to the existing method [6]. Zheng Danyang et al (2017) has proposed using a self-adaptive quantum revolving door update mode --- self-adaptive quantum genetic algorithm (SAQGA) for solving DVRP problem under the minimum distribution cost [7]. Liu Qing (2013) has studied the route problem of vehicle picking up and delivering goods simultaneously which had the stochastic demand[8] ; 
Wang Xiaobo and Li Yijun (2007) have studied the scheduling of vehicle with time window under E-commerce mode and adopted modified two-stage algorithm for solution [9]. Li Kunpeng and Ma Shihua (2008) have studied the transportation coordination and scheduling in the supply chain dominated by 3PL under JIT background [10]. Most literatures and most researches base on the uniform vehicle model, takes the minimum operation cost or shortest driving distance as the target without considering the difference of loading capacity and transportation cost of vehicle. Based on genetic algorithm, the paper considers the impact of the loading capacity and transportation cost of vehicle on the transportation expenses, solves the vehicle scheduling and line selection program of different models and verifies the validity of the model.

A fresh food processing company in a city needs to conduct fresh product transportation within the range of the entire city, and the transportation route of fresh products is from the processing factory to the 5 depots distributed in the city via trucking at first and then from the depot to the 20 customers distributed within the city; see Table 1.

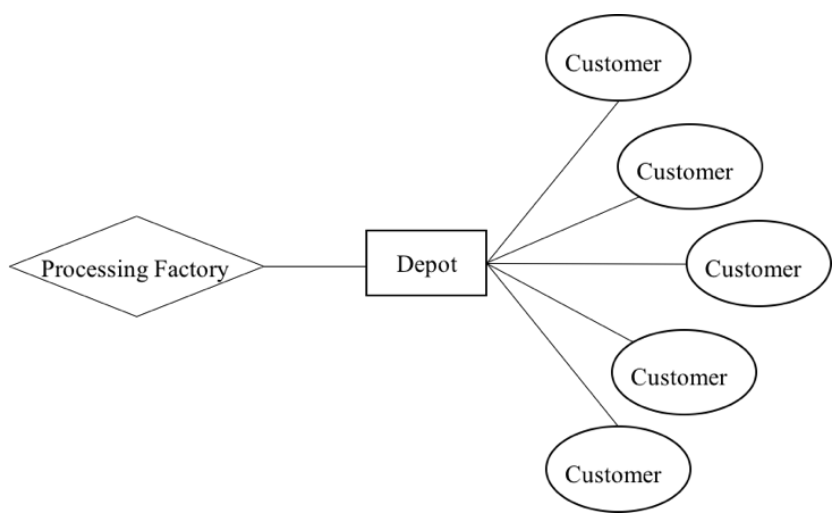

Fig. 1 Two-node transport diagram

Mathematical model:

$$
\min \mathrm{Z}=\sum_{\mathrm{i}=1}^{\mathrm{I}} \sum_{\mathrm{h}_{\mathrm{i}}=1}^{\mathrm{H}_{\mathrm{i}}} \mathrm{V}_{\mathrm{h}_{\mathrm{i}}} \mathrm{X}_{\mathrm{h}_{\mathrm{i}}} \mathrm{Y}_{\mathrm{i}}+\sum_{\mathrm{g}=1}^{\mathrm{G}} \mathrm{C}_{\mathrm{mg}} \mathrm{S}_{\mathrm{mg}} \mathrm{X}_{\mathrm{mg}}+\sum_{\mathrm{g}=1}^{G} \sum_{\mathrm{k}=1}^{\mathrm{K}} \mathrm{C}_{\mathrm{gk}} \mathrm{S}_{\mathrm{gk}} \mathrm{X}_{\mathrm{gk}}+\sum_{\mathrm{g}=1}^{\mathrm{G}} \sum_{\mathrm{k}=1}^{\mathrm{K}} \mathrm{X}_{\mathrm{gk}} \mathrm{l}_{\mathrm{gk}}
$$

s.t

$$
\begin{aligned}
& \sum_{\mathrm{k}=1}^{\mathrm{K}} \mathrm{S}_{\mathrm{kg}}=\mathrm{S}_{\mathrm{mg}} \\
& \sum_{\mathrm{g}=1}^{\mathrm{G}} \sum_{\mathrm{k}=1}^{\mathrm{K}} \mathrm{S}_{\mathrm{kg}} \leq \operatorname{Cap}_{\mathrm{g}} \quad(\mathrm{g}=1,2, \cdots, \mathrm{G}) \\
& \sum_{\mathrm{g}=1}^{\mathrm{G}} \mathrm{X}_{\mathrm{gk}}=1 \\
& \mathrm{X}_{\mathrm{hi}} \in\{0,1\} \quad\left(\mathrm{h}_{\mathrm{i}}=1,2, \cdots, \mathrm{H}_{\mathrm{i}} . \mathrm{i}=1,2, \cdots, \text { I. }\right) \\
& \mathrm{X}_{\mathrm{gk}} \in\{0,1\} \quad(\mathrm{g}=1,2, \cdots, \mathrm{G} . \mathrm{k}=1,2, \cdots, \mathrm{K} .) \\
& \mathrm{Y}_{\mathrm{i}} \leq \mathrm{H}_{\mathrm{i}}(\mathrm{i}=1,2, \cdots, \quad \text { I. }) \\
& \mathrm{X}_{\mathrm{mg}} \in\{0,1\} \quad(\mathrm{g}=1,2, \cdots, \mathrm{G} .)
\end{aligned}
$$




$$
\begin{gathered}
\mathrm{d}_{\mathrm{kg}} \leq \sum_{\mathrm{i}=1}^{\mathrm{I}} \sum_{\mathrm{h}_{\mathrm{i}}=1}^{\mathrm{H}_{\mathrm{i}}} \operatorname{Cap}_{\mathrm{i}} \mathrm{Y}_{\mathrm{i}} \mathrm{X}_{\mathrm{h}_{\mathrm{i}}} \\
\mathrm{d}_{\mathrm{kg}}=\mathrm{S}_{\mathrm{gk}}(\mathrm{g}=1,2, \cdots, \mathrm{G} . \mathrm{k}=1,2, \cdots, \mathrm{K} .)
\end{gathered}
$$

Where:

I : Number of vehicle type;

i : Index of vehicle type $, \mathrm{i}=1,2, \cdots, \mathrm{I}$;

$\mathrm{H}_{\mathrm{i}}$ : Number of the $\mathrm{i}$ 'th vehicle type;

$\mathrm{h}_{\mathrm{i}}$ : Index of vehicle of type $\mathrm{i}, \mathrm{i}=1,2, \cdots, \mathrm{I}$;

$\mathrm{V}_{\mathrm{h}_{\mathrm{i}}}$ : Fix cost of vehicle $\mathrm{h}_{\mathrm{i}}, \mathrm{h}_{\mathrm{i}}=1,2, \cdots, \mathrm{H}_{\mathrm{i}}$;

$\mathrm{X}_{\mathrm{h}_{\mathrm{i}}}$ : If the vehicle $\mathrm{h}_{\mathrm{i}}$ is used to be deliver the goods, it take value 1 , otherwise, it takes value 0 , which is the decision variable, $h_{i}=1,2, \cdots, H_{i}$;

$\mathrm{Y}_{\mathrm{i}}$ : Used vehicle type his number; M: producer; G: Number of depot; $\mathrm{g}$ : index of depot , $\mathrm{g}=1,2, \cdots$, $\mathrm{G}$;

$\mathrm{C}_{\mathrm{mg}}$ : Unit freight of good from $\mathrm{m}$ to g. $(\mathrm{m}=1,2, \cdots$, M. $\mathrm{g}=1,2, \cdots, \mathrm{G})$;

$\mathrm{S}_{\mathrm{mg}}$ : Freight volume from $\mathrm{m}$ to $\mathrm{g}$. $(\mathrm{m}=1,2, \cdots, \mathrm{M} . \mathrm{g}=1,2, \cdots, \mathrm{G})$;

$\mathrm{X}_{\mathrm{mg}}$ : If one of the $\mathrm{m}$ to $\mathrm{g}$ is chosen to transport the goods it take value 1 , otherwise, it takes value 0 , which is the decision variable,$(\mathrm{m}=1,2, \cdots, \mathrm{M} . \mathrm{g}=1,2, \cdots, \mathrm{G})$;

$\mathrm{K}$ : Number of customer; $\mathrm{k}$ : Index of customer , $\mathrm{k}=1,2, \cdots, \mathrm{K}$;

$\mathrm{X}_{\mathrm{gk}}$ :If vehicle pass one of $\mathrm{g}$ to $\mathrm{k}$, it take value 1 , otherwise, it takes value 0 , which is the decision variable. $(\mathrm{g}=1,2, \cdots, \mathrm{G} . \mathrm{k}=1,2, \cdots, \mathrm{K})$;

$\mathrm{l}_{\mathrm{gk}}$ :Distance between depot $\mathrm{g}$ and customer $\mathrm{k} .(\mathrm{g}=1,2$, $\cdots, \mathrm{G} . \mathrm{k}=1,2, \cdots, \mathrm{K})$;

$\mathrm{d}_{\mathrm{kg}}$ : Demand of customer k for depot $\mathrm{g}$;

$\mathrm{S}_{\mathrm{gk}}$ : supply of depot $\mathrm{g}$ to customer $\mathrm{k}$;

Cap $_{\mathrm{i}}$ : Capacity of vehicle type $\mathrm{i}$.

\section{Mathematical Model}

Genetic Algorithm seeks the optimal solution through adaptive random iterations by simulating the evolutionary laws of Darwin Evolution (survival of the fittest, survival of the fittest) and the genetic mechanism of Mendel genetics.

Genetic Algorithm uses the principles of simulation genetics and natural selection, and through the mechanisms of natural selection, heredity and mutation, the improvement of individual adaptability is gradually accomplished. In a sense, GA is a mathematical simulation of the process of biological evolution, which embodies the principle of survival of the fittest in nature. Similarly, GA knows nothing about the nature of the problem, starting with a population that represents a potential solution to a problem, and each population is made up of a certain number of individuals encoded by the gene. In fact, each individual is a chromosome with a characteristic entity, the solution of the problem is expressed by chromosomes. The selection of chromosomes based on an adaptive value. The Genetic Algorithm only needs to evaluate each chromosome produced by the algorithm, and promote the adaptive ability to obtain more breeding opportunities. In the process of calculation, the coding string structure is generally binary, and the value of each position corresponds to the corresponding allele. By compiling a group of chromosomes that are hypothetical solutions and placing it in the "environment" 
of the problem, the corresponding adaptive function is evaluated. According to certain principles, select the chromosomes that can adapt to this environment to replicate, eliminate the less adaptive individuals, and then through the intersection, mutation process to produce the offspring chromosome group, which is more able to adapt to the environment, and finally continue to use the next round to screen, until the most suitable value for the environment appears.

\subsection{Coding and Decoding}

The coding process includes the allocation of the distribution relationship between the logistics nodes and the customers and the routes of vehicles. Firstly, about the distribution relationship between the logistics nodes and the customers, three-layer coding is adopted. Suppose there are $\mathrm{n}$ customers and $\mathrm{m}$ logistics nodes. At the first layer disrupt the order of $\mathrm{n}$ customers at arbitrary; at the second layer disrupt the order of $\mathrm{m}$ logistics nodes at arbitrary; at the third layer generate [1, m-1] different numbers between 1 and n-1 as the nodes. Three-layer coding is finished.

Suppose $\mathrm{m}=10$ and $\mathrm{n}=5$, then the first coding example is:

$$
\begin{array}{lllllllll|lll|ll}
5 & 7 & 3 & 4 & 6 & 8 & 9 & 10 & 2 & 2 & 3 & 1 \mid 2 & 8
\end{array}
$$

The meaning of the individual is: Disrupt at Position 2 and Position 8 in the first-layer coding, i.e., all the customers are divided into three parts: $(1,5),(7,3,4,6,8,9)$ and $(10,2)$; in total three nodes are needed, and according to the second-layer coding, they are $(2,3,1)$; that means the three groups of customers are served by 2,3 and 1 logistics nodes respectively.

Then it is the dispatch of transport vehicles. Disrupt vehicles at arbitrary and then dispatch them onto the route from the production factory to logistics nodes and then to customers; the corresponding relations is: vehicles correspond to above-mentioned production factory to No.2, No.3 and No.1 logistics nodes, and then it is from No. 2 logistics node to No.1 customer, No.5 customer, and the rest can be done in the same way.

After finishing the distribution at that stage, according to the demand of each node, the product demand of each logistics node can be calculated; meanwhile due to natural number coding, the serial number after coding can be converted into the specific solution of problems.

\subsection{Genetic Operator}

The article adopts Parthenon-Genetic Algorithm (called PGA in brief) to realize problem solving. During problem solving use the coding and decoding methods mentioned above. PGA is a genetic method using and choosing gene transposition, gene shifting and gene inversion for offspring reproduction. Therein, gene transposition operator is the process to change the genes at some positions in a chromosome according to certain probability Pe; the position being changed is arbitrary. Gene transposition can be divided into single gene transposition and multiple gene transposition. Single gene transposition is to change the positions of a pair of genes (two genes) only at one time; multiple gene transposition is for a given positive integer Ue, to choose an arbitrary number $\mathrm{i}(1 \leq \mathrm{i} \leq \mathrm{Ue})$ and change $\mathrm{i}$ pairs of genes at a time. Single gene transposition is also called mutation operator; due to the coding method is natural number coding and repeated number is not allowed, the transposition adopts the method to change the position of a gene and the position of the other gene with the same number. The operation is shown in Figure 2.

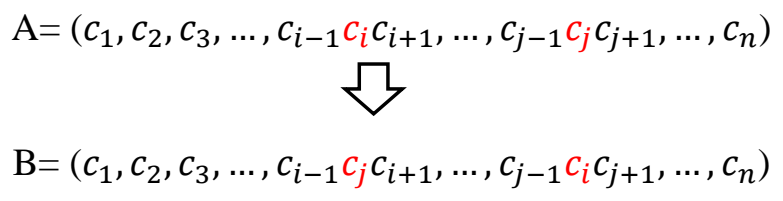

Fig 2 Single Gene Transposition Operation

Gene shifting operator is to shift the genes in some substrings in a chromosome to the back successively according to certain probability Ps and shift the last gene in the substring to the headmost. In a chromosome, the 
substring to which the gene shifting is conducted and its length are chosen arbitrarily. Gene shifting can be divided into single gene shifting and multiple gene shifting. Single gene shifting means to choose only a substring in a chromosome to conduct gene shifting while multiple gene shifting is for a given positive integer in advance, to choose an arbitrary number $\mathrm{j}(1 \leq \mathrm{j} \leq \mathrm{Uj})$ and then to choose $\mathrm{j}$ substrings in a chromosome to conduct gene shifting. Shifting operation must satisfy a condition that it shall not lead to the overflow of the new parent node's volume; the single gene shifting operation is in Figure 3.

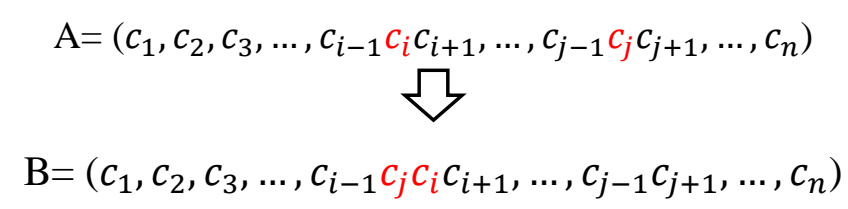

Fig 3 Single Gene Shifting Operation

Gene inversion operator is to inverse the genes in some substrings in a chromosome according to certain probability Pi successively, and in a chromosome the substrings to which gene inversion is conducted and their length are chosen arbitrarily. Gene inversion can be divided into single gene inversion and multiple gene inversion. Single point inversion means to choose only a substring in a chromosome to conduct gene inversion while multiple gene inversion is for a given positive integer in advance, to choose an arbitrary number $\mathrm{j}(1 \leq \mathrm{j} \leq \mathrm{Uj})$ and then to choose $\mathrm{j}$ substrings in a chromosome to conduct gene inversion. Inversion operator does not need to judge the overflow situation of the parent node's volume; the operation is in Figure 4.

$$
\begin{aligned}
& \mathrm{A}=\left(c_{1}, c_{2}, c_{3}, \ldots, c_{i-1} c_{i} c_{i+1}, \ldots, c_{j-1} c_{j} c_{j+1}, \ldots, c_{n}\right) \\
& \mathrm{B}=\left(c_{1}, c_{2}, c_{3}, \ldots, c_{i-1} c_{j} c_{j-1}, \ldots, c_{i+1} c_{i} c_{j+1}, \ldots, c_{n}\right)
\end{aligned}
$$

Fig 4 Single Point Inversion

Multiple genetic operator is usually used when the chromosome string length 1 is big while single genetic operator is used when 1 is small. On such basis, the article uses the Genetic Algorithms including single gene transposition, single gene shifting and single gene inversion for offspring production to carry out genetic operator operation.

\section{3 choose}

Adopt the strategy that elite individuals shall be kept upon the choosing operation; copy the individual with the highest fitness function to the next generation directly. After all the parent generation individuals finish the genetic operator, adopt the mechanism that elite individuals shall be kept again; replace the individuals with the worst fitness function in the new group with the elite individuals before the genetic operator operation and get rid of low-quality individuals to make elite individuals continue.

\subsection{Loop Iteration}

Determine if the terminal conditions are reached; stop computing if it satisfies the conditions, and otherwise go on the iterative computation till the terminal conditions are reached. 


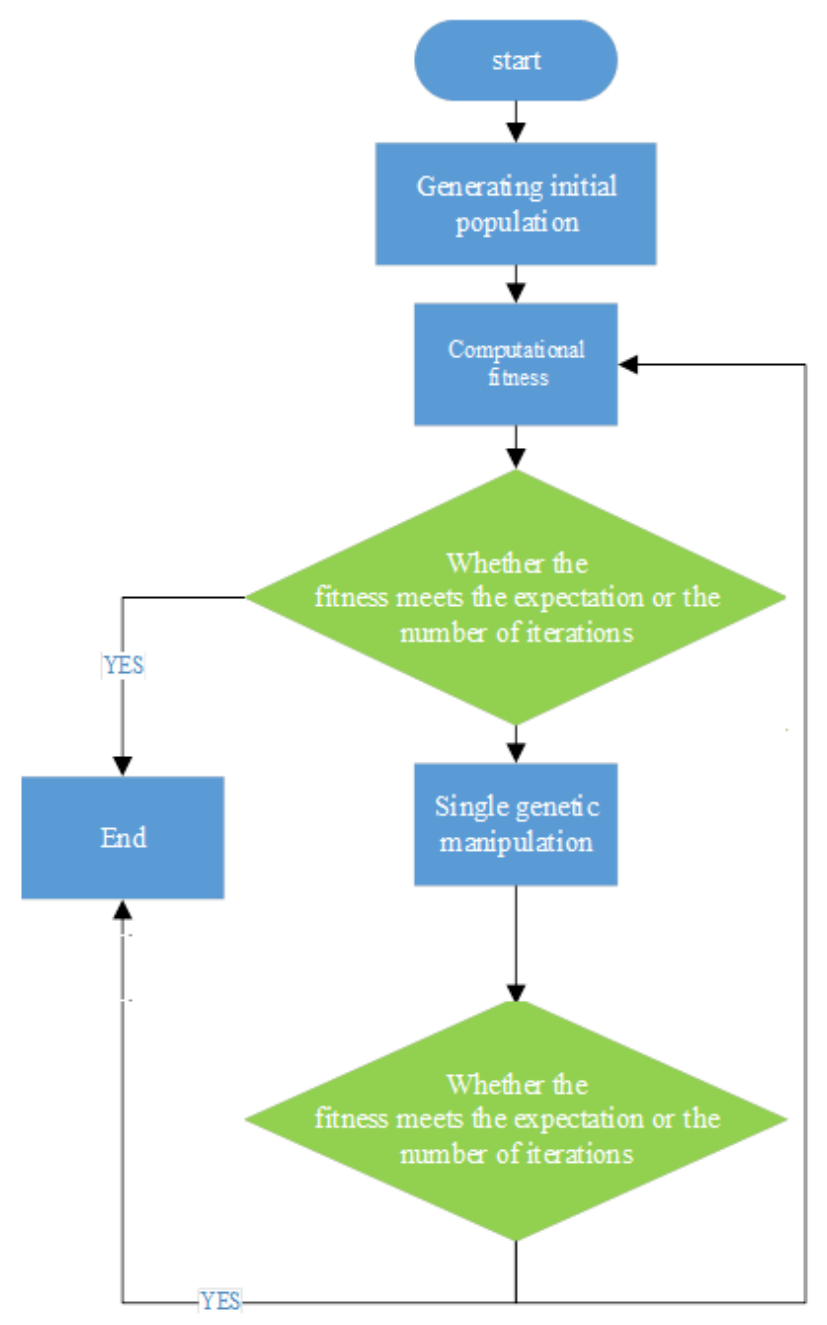

Algorithm flow chart

\section{Example}

A fresh food processing company in a city needs to conduct fresh product transportation within the range of the entire city, and the transportation route of fresh products is from the processing factory to the 5 depots distributed in the city via trucking at first and then from the depot to the 20 customers distributed within the city; see Table 1.

The company has 25 trucks in total, among which there are 4 trucks whose maximum carrying capacity is $3 \mathrm{t}$, and the freight is $1.8 / \mathrm{km}$; there are 12 trucks whose maximum carrying capacity is $6 \mathrm{t}$ and the freight is $2 / \mathrm{km}$; there are 9 trucks whose maximum carrying capacity is $8 \mathrm{t}$ and the freight is $2.4 / \mathrm{km}$; they are responsible for the straight-line transportation of 25 routes respectively. 
TABLE 1: Position Information

\begin{tabular}{|c|c|c|c|}
\hline \multirow{2}{*}{ No. } & \multirow{2}{*}{ Category } & \multicolumn{2}{|c|}{ Relative Position } \\
\hline & & $\mathrm{x}$ Coordinate & y Coordinate \\
\hline Processing Factory & Processing Factory & 50 & 50 \\
\hline Depot 1 & \multirow{5}{*}{ Depot } & 47 & 71 \\
\hline Depot 2 & & 34 & 37 \\
\hline Depot 3 & & 63 & 64 \\
\hline Depot 4 & & 56 & 70 \\
\hline Depot 5 & & 57 & 28 \\
\hline Customer 1 & \multirow{20}{*}{ Customer } & 42 & 80 \\
\hline Customer 2 & & 13 & 11 \\
\hline Customer 3 & & 34 & 95 \\
\hline Customer 4 & & 62 & 56 \\
\hline Customer 5 & & 28 & 76 \\
\hline Customer 6 & & 69 & 84 \\
\hline Customer 7 & & 11 & 37 \\
\hline Customer 8 & & 21 & 22 \\
\hline Customer 9 & & 63 & 90 \\
\hline Customer 10 & & 24 & 79 \\
\hline Customer 11 & & 32 & 42 \\
\hline Customer 12 & & 96 & 76 \\
\hline Customer 13 & & 41 & 79 \\
\hline Customer 14 & & 66 & 28 \\
\hline Customer 15 & & 54 & 56 \\
\hline Customer 16 & & 17 & 11 \\
\hline Customer 17 & & 12 & 74 \\
\hline Customer 18 & & 83 & 58 \\
\hline Customer 19 & & 46 & 28 \\
\hline Customer 20 & & 44 & 16 \\
\hline
\end{tabular}

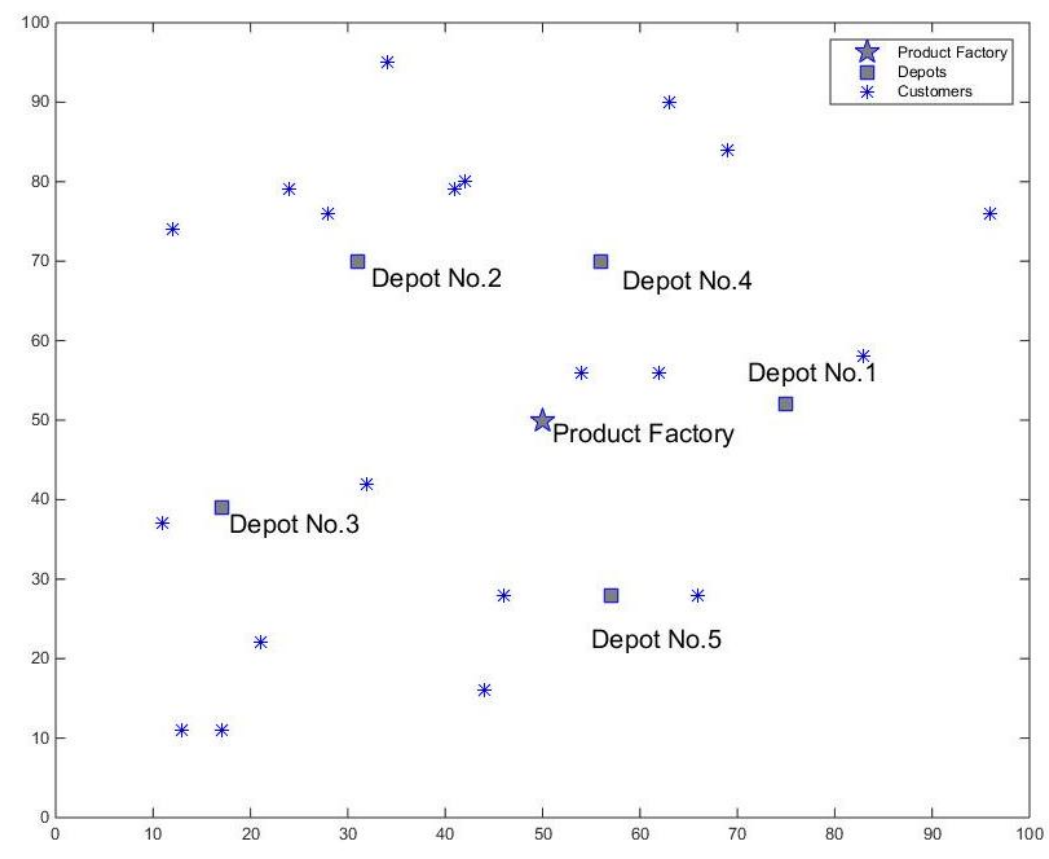

Fig 5 Factory Location Diagram 
Use straight-line distance to simulate the distance between the processing factory, the depot and customers; do not take into consideration of the influence of traffic jams and traffic lights etc.

The production of the processing factory proceeds based on customers' orders at each month, i.e., the processing factory is capable of satisfying customers' needs and the capacity of the depot can satisfy product transfer need. Customers' demand data is shown in Table II.

TABLE II: Data on Customers' Demand

\begin{tabular}{|c|c|c|c|}
\hline No & Quantity Required(t) & No. & Quantity Required(t) \\
\hline Customer 1 & 16 & Customer 11 & 28 \\
\hline Customer 2 & 15 & Customer 12 & 13 \\
\hline Customer 3 & 18 & Customer 13 & 19 \\
\hline Customer 4 & 27 & Customer 14 & 10 \\
\hline Customer 5 & 26 & Customer 15 & 10 \\
\hline Customer 6 & 19 & Customer 16 & 28 \\
\hline Customer 7 & 28 & Customer 17 & 16 \\
\hline Customer 8 & 12 & Customer 18 & 19 \\
\hline Customer 9 & 19 & Customer 19 & 26 \\
\hline Customer 10 & 15 & Customer 20 & 26 \\
\hline
\end{tabular}

Goal: Optimal vehicle distribution route and route-matching vehicles.

Take advantage of MATLAB program to realize the Genetic Algorithm of above-mentioned problem. Parameter Setting: the maximum crossover probability is 0.9 and the maximum mutation probability is 0.1 . The population size of each generation is 200, and the evolution result after a cycle of 200 generations is shown by Figure 6 and Figure 7. The minimum expenses is 10434.

Comparing traditional genetic algorithm and single genetic algorithm, it is seen single parent genetic algorithm converges at 42th generation, but traditional genetic algorithm reaches convergence after 100 generations. It proves the effectiveness of single parent genetic algorithm.

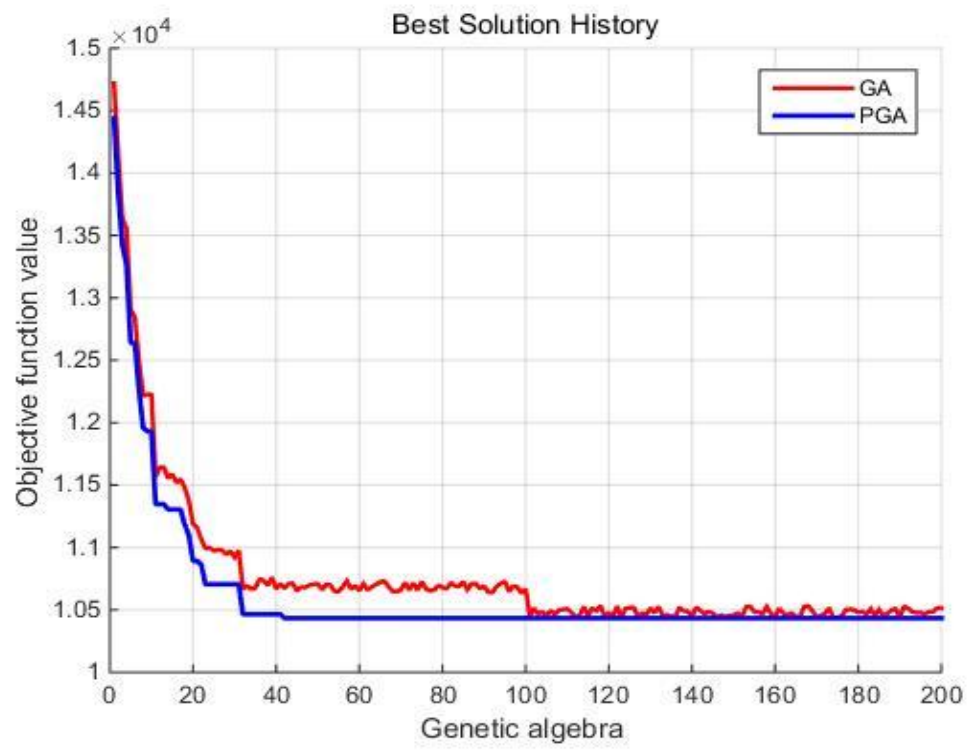

Fig 6 Diagram of the Fitness Function Changing with the Genetic Algebra 


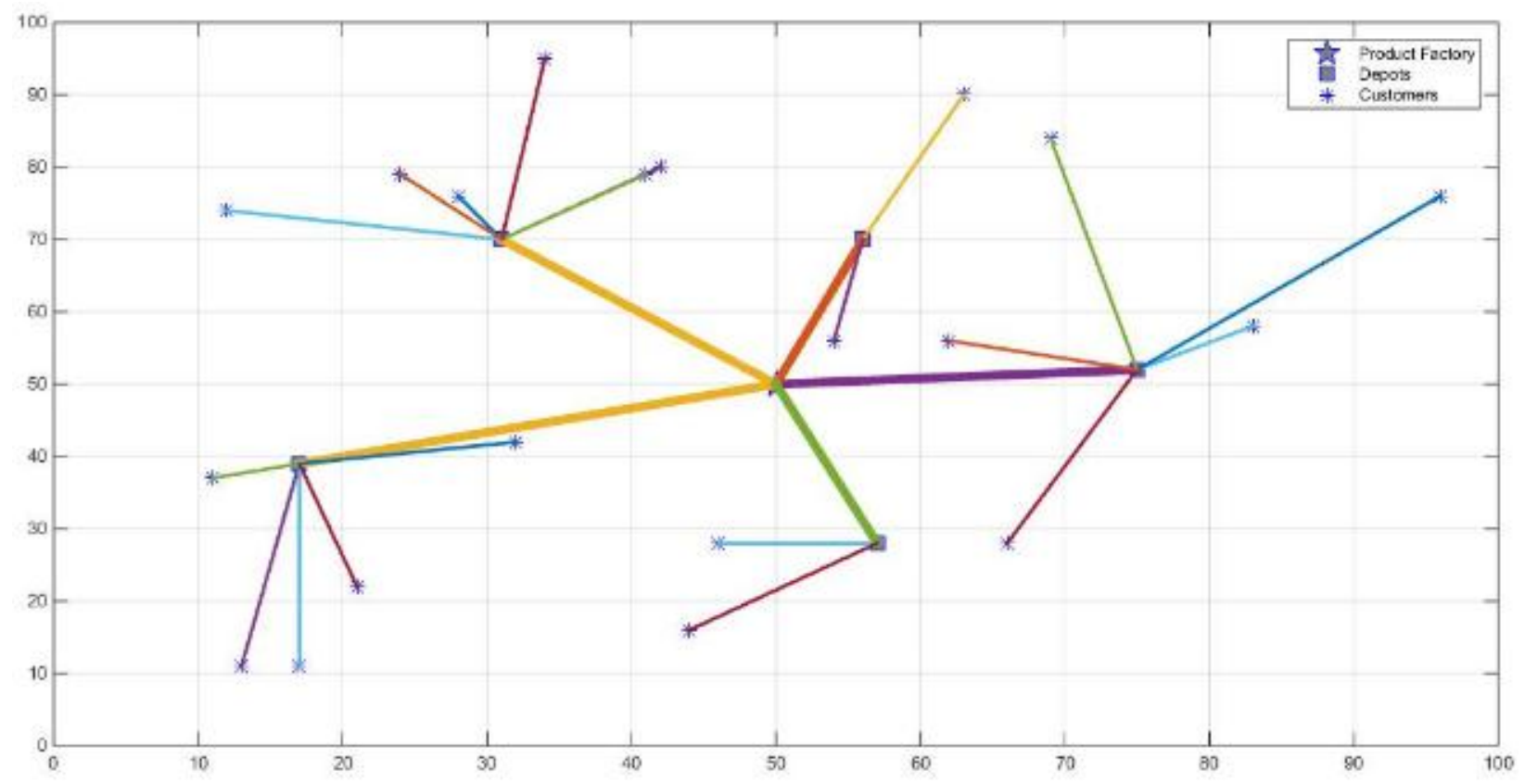

Fig 7 Vehicle Dispatch Diagram

TABLE 3 Table on the Vehicle Dispatch from depot to customers

\begin{tabular}{|c|c|}
\hline Depot No. & Customer No. \\
\hline Depot 1 & $\begin{array}{lllll}6 & 18 & 14 & 12 & 4\end{array}$ \\
\hline Depot 2 & $\begin{array}{llllll}1 & 13 & 17 & 3 & 5 & 10\end{array}$ \\
\hline Depot 3 & $\begin{array}{lllll}2 & 7 & 16 & 8 & 11\end{array}$ \\
\hline Depot 4 & $9 \quad 15$ \\
\hline Depot 5 & 1920 \\
\hline
\end{tabular}

Vehicles are numbered 1, 21, 8, 22, 19, 7, 16, 14, 5, 3, 24, 25, 11, 17, 18, 10, 20, 9, 23, 6, 15, 12, 4, 13, and 2, responsible for the vehicle transportation from the processing factory to three depots and then from depot 1 , depot 2, depot 3, depot 4 and depot 5 to respective customers; the numbering of customers is done in the same way mentioned above. Therein, 1-4 mean 3t vehicles; 5-16 mean 6t vehicles and others are $8 t$ vehicles.

From the figure, it can be known that the algorithm has advantages as follows: it can effectively solve the dispatch of different vehicles during transportation, and the distribution of vehicles for different routes has a big influence on the overall freight; the algorithm converges fast. For the transportation line and vehicle dispatch problems based on Genetic Algorithm, the model and problem-solving algorithm used in this article are quite effective.

\section{Conclusion}

The article simplifies the problem of dual-node transportation to a transportation model; based on Genetic Algorithm, taking the optimization of the transportation expenses of the bi-level logistics node of the sea food in a city as the optimization goal, conduct real number encoding to vehicles and the routes and use crossover and mutation operations for problem solving; the result demonstrates the method adopted by the problem can excellently settle the problem of dual-node transportation and it is effective. 


\section{References}

[1] Dantizig, G.,Ramser, J1. The truck dispatching problem [J] . Management Science, 1959, 6(1): 80-911

[2] Branke J, Middendorf M, Noeth G, Dessouky M. Waiting strategies for dynamic vehicle routing[J]. Transportation Science 2005, 39:298-312.

[3] Li J Q, Borenstein D, MirchandaniP B.A decision support system for the single-depot vehicle scheduling probIem[J]. Computers \& Operations Research, 2007, 34(4):1008-1032.

[4] Wang Liang, Li Shixun, Sun Shaorong. Research on Integration and Optimization of the Direct Despatching Transportation Based on Stochastic Demand and Inventory [J]. Management Engineering Journal, 2007, 21 (2): 130-133.

[5] Yang Yang, Wang Long. Real-Time Scheduling Model and Coevolution Genetic Algorithm of Mobile Supply Chain [J]. Nanjing University of Science and Technology Journal (natural science), 2011, 35(4):579-584.

[6] Wang Xu, Ge Xianlong. Research on Dynamic Vehicle Scheduling of Solution Algorithm Based on Two Stage [J]. Control and Decision, 2012, 27(2): 175-181.

[7] Zheng Danyang, Mao Jianlin, et al. Self-Adaptive Quantum Genetic Algorithm of Solving Scheduling Problem of Vehicle with Dynamic Demand [J]. Sensor and Microsystem, 2017, 36(8): 130-133.

[8] Liu Qing, Research on Modeling and Optimization of Routing Problem of Vehicle Picking Up and Delivering Goods Simultaneously with Scholastic Demand [D]. Nanjing University of Aeronautics and Astronautics: Nanjing University of Aeronautics and Astronautics, 2013.

[9] Wang Xiaobo, Li Yijun. Optimization of Vehicle Dispatch with Time Window based on Improved Two-stage Algorithm under E-commerce [J], Chinese Journal of Management Science, 2007,15(6):52-59.

[10] Li Kunpeng, Ma Shihua. 3PL Transport Coordination \& Dispatch Problem Modeling and Analysis based on JIT Distribution [J], Chinese Journal of Management Science, 2008,16(1):73-79. 